\title{
High negative valence does not protect emotional event-related potentials from spatial inattention and perceptual load
}

\author{
Stefan Wiens • Tanaz Molapour • Judith Overfeld • \\ Anders Sand
}

Published online: 18 November 2011

(C) Psychonomic Society, Inc. 2011

\begin{abstract}
Previous research suggests that intense, emotional pictures at fixation elicit an early posterior negativity (EPN) and a late positive potential (LPP) despite manipulations of spatial inattention and perceptual load. However, if high emotional intensity protects against such manipulations, then these manipulations should reduce emotional effects on EPN and LPP more strongly for medium than for intense emotional pictures. To test this prediction, pictures that were high negative, medium negative, or neutral were shown at fixation, and a small letter string was superimposed on the picture center. When participants attended the pictures, there were clear emotional effects on EPN and LPP. When participants attended the letter string, the emotional effects on LPP decreased; this decrease was smaller for medium than for high negative pictures. Thus, opposite of predictions, spatial inattention reduced the emotional effects more strongly for high than for medium negative pictures. As a manipulation of perceptual load, participants performed the letter task with one, three, or six relevant letters. Irrespective of load, EPN and LPP were similar for high and medium negative pictures. Our findings suggest that high negative valence does not protect EPN and LPP more strongly from effects of spatial inattention and perceptual load than does medium negative valence.
\end{abstract}

Keywords Attention · Perceptual load · Event-related potentials $\cdot$ Early posterior negativity $\cdot$ Late positive potential

S. Wiens $(\bowtie) \cdot$ T. Molapour $\cdot$ J. Overfeld $\cdot$ A. Sand Department of Psychology, Stockholm University, Frescati Hagväg 9,

10691 Stockholm, Sweden

e-mail: sws@psychology.su.se
Emotion guides attention (Peira, Golkar, Larsson, \& Wiens, 2010; Vuilleumier \& Huang, 2009), but to be effective, emotion needs to capture attention even if it is currently focused on other stimuli. In one approach to studying the degree to which emotion can capture attention, participants perform a task in the context of task-irrelevant, emotional pictures at task-irrelevant locations. If the emotional pictures attract attention, then one could infer that emotion is processed even when attention is directed toward another task and location. In support of this idea, brain imaging studies suggest that when emotional faces or emotional pictures are shown and the main task is rather simple, the task-irrelevant emotional pictures are processed (e.g., elicit amygdala activation) even if they are shown in the periphery (Pessoa, 2005; Pessoa, Padmala, \& Morland, 2005; Silvert, Lepsien, Fragopanagos, Goolsby, Kiss, \& Taylor, 2007; Vuilleumier, 2005; Vuilleumier, Armony, Driver, \& Dolan, 2001). For example, when participants attended two houses to the left and right of the fixation point while task-irrelevant faces were shown above and below the fixation point (or vice versa), results showed greater amygdala activation to fearful than to neutral faces, even if the faces were unattended (Vuilleumier et al., 2001).

In event-related potential (ERP) studies of this subject, processing of emotion was mostly indexed by ERPs that differed between emotional and neutral pictures, irrespective of their valence. The ERP difference waves between emotional and neutral pictures showed an early posterior negativity (EPN) and a late positive potential (LPP) (Schupp, Flaisch, Stockburger, \& Junghöfer, 2006). Emotional words also elicited the EPN and LPP (Kissler, Herbert, Winkler, \& Junghöfer, 2009). The EPN refers to a bilateral temporaloccipital negativity that occurs about $200 \mathrm{~ms}$ after picture onset and that originates in the occipital cortex with parietal contributions (Junghöfer, Bradley, Elbert, \& Lang, 2001). 
The EPN appears to reflect a call for attentional resources because it resembles the selection negativity observed in studies of directed attention (Codispoti, Ferrari, De Cesarei, \& Cardinale, 2006). The LPP is a central-parietal positivity that occurs about $300 \mathrm{~ms}$ after picture onset (Cuthbert, Schupp, Bradley, Birbaumer, \& Lang, 2000; Palomba, Angrilli, \& Mini, 1997). The LPP is associated with activations in lateral occipital, inferotemporal, and parietal visual areas (Sabatinelli, Lang, Keil, \& Bradley, 2007). The traditional P300 or P3b peak (Sutton, Braren, Zubin, \& John, $1965)$ is similar to the LPP in that both peaks index the allocation of capacity-limited, attentional resources to salient events (Bradley, 2009; Hajcak, MacNamara, \& Olvet, 2010; Olofsson, Nordin, Sequeira, \& Polich, 2008). Although the LPP can last several seconds, research suggests that later slow wave responses represent processes different from the initial response that occurs around $300 \mathrm{~ms}$ (Foti, Hajcak, \& Dien, 2009). In sum, the EPN and LPP are promising ERP indexes of emotional effects on attention. Because emotional stimuli have intrinsic relevance for the organism, the emotional effects on attention have been referred to as motivated attention (Schupp et al., 2006).

Several ERP studies have suggested that EPN and LPP to emotional versus neutral pictures are eliminated when pictures are spatially unattended (De Cesarei, Codispoti, \& Schupp, 2009; Eimer, Holmes, \& McGlone, 2003; Holmes, Kiss, \& Eimer, 2006; Holmes, Vuilleumier, \& Eimer, 2003; MacNamara \& Hajcak, 2009). For example, when each trial showed a vertical pair of faces and a horizontal pair of houses (or vice versa) (cf. Vuilleumier et al., 2001), the LPP to fearful faces was eliminated when the faces were unattended (Holmes et al., 2003). Also, the LPP to emotional faces was eliminated when the faces were shown in the periphery and participants performed a line task at fixation (Eimer et al., 2003). The LPP was even eliminated when faces were shown at fixation and participants performed a simple one-back task on the bars to the left and right of the faces (Holmes et al., 2006). Further, the EPN and LPP responses to arousing, emotional pictures from the International Affective Picture Set (IAPS; Lang, Bradley, \& Cuthbert, 2008) were eliminated if these pictures were shown in the periphery and were unattended (De Cesarei et al., 2009; MacNamara \& Hajcak, 2009, 2010). Last, when IAPS pictures were shown in large format $\left(40^{\circ}\right.$ visual angle) and spatial attention was directed to neutral areas within the pictures, emotional effects on the LPP were eliminated (Dunning \& Hajcak, 2009; Hajcak, Dunning, \& Foti, 2009). Taken together, these findings suggest that emotional effects on EPN and LPP are eliminated when emotional pictures or emotional areas within the pictures are not spatially attended.

However, recent evidence suggests that emotional pictures at fixation may have residual emotional effects on EPN and LPP, even if these pictures are apparently not spatially attended. First, when pictures of spiders and mushrooms were shown at fixation and participants performed a task on letters that surrounded the pictures, spider-fearful participants showed a greater LPP to spiders versus mushrooms than did nonfearful participants, even though the pictures were unattended (Norberg, Peira, \& Wiens, 2010). Second, when mutilated bodies were shown at fixation and participants performed a matching task on bars to either side of the pictures, there was an emotional effect on LPP when participants believed that the pictures were real (Mocaiber, Pereira, Erthal, Machado-Pinheiro, David, \& Cagy, 2010). Third, when negative and neutral IAPS pictures were shown at fixation and participants performed a task on letters that surrounded the pictures, there was an emotional effect on LPP but not on EPN (Wiens, Sand, Norberg, \& Andersson, 2011). Fourth, in a similar study with negative and neutral IAPS pictures that depicted only simple figures, there was nonetheless an emotional effect on both EPN and LPP (Sand \& Wiens, 2011).

Indeed, recent evidence suggests that emotional pictures at fixation have residual emotional effects on EPN and LPP despite high levels of perceptual load (Norberg et al., 2010; Sand \& Wiens, 2011). As theorized by Lavie $(1995,2010)$, perceptual load refers to the extent to which attentional resources are depleted. If these resources are depleted, then task-irrelevant stimuli are no longer processed. So, if a main task involves a large number of items that need to be processed, then task-irrelevant, emotional distracters should no longer be processed because attentional resources are depleted by the main task (perceptual load). Notably, Lavie emphasized that perceptual load differs from cognitive load in that perceptual load acts on early perceptual processes. For example, Lavie stipulated that a working memory load might increase perceptual processing, whereas a perceptual load would decrease perceptual processing. Therefore, the effects of perceptual load on emotional ERPs might differ from those of cognitive load imposed by manipulations such as task difficulty (Hajcak, Dunning, \& Foti, 2007) or working memory load (Holmes, Nielsen, Tipper, \& Green, 2009; MacNamara, Ferri, \& Hajcak, 2011).

In the first of two studies that found residual emotional effects even under high perceptual load (Norberg et al., 2010), spider-fearful participants viewed spiders and mushrooms that were surrounded by a letter ring with either three or six letters. Critically, emotional effects on LPP were unaffected by whether perceptual load was low (three letters) or high (six letters). Similarly, when participants performed a letter discrimination task on letters that surrounded negative and neutral IAPS at fixation (Sand \& Wiens, 2011), perceptual load was manipulated by increasing the number of letters from one to three. The results showed that emotional effects on EPN and LPP remained significant despite high perceptual load. 
These findings suggest that residual emotional effects on EPN and LPP to task-irrelevant, emotional pictures may be observed despite manipulations of spatial attention and perceptual load if the pictures are shown at fixation. However, this conclusion is challenged by the finding that the LPP to fearful faces at fixation was eliminated when the faces were unattended (Holmes et al., 2006). A reasonable explanation for this discrepancy between findings is that emotional effects on EPN and LPP may be more persistent for emotional pictures than for emotional faces because of the pictures' greater emotional intensity. Indeed, emotional pictures are more arousing than emotional faces (Britton, Taylor, Sudheimer, \& Liberzon, 2006), and emotional effects on EPN and LPP increase with arousal (Cuthbert et al., 2000; Schupp, Junghöfer, Weike, \& Hamm, 2003; Wiens, Sand, \& Olofsson, 2011). The evidence is consistent with this explanation because, irrespective of inattention and perceptual load, residual emotional effects on EPN and LPP were found for emotional IAPS (Sand \& Wiens, 2011; Wiens, Sand, Norberg, \& Andersson, 2011), emotional pictures that participants believed to be real scenes (Mocaiber et al., 2010), and spiders in participants with spider fear (Norberg et al., 2010).

Taken together, the research shows that despite manipulations of spatial attention and perceptual load, emotional effects on EPN and LPP remain significant to intense, emotional pictures at fixation. These findings suggest that the high emotional intensity protects against manipulations of spatial attention and perceptual load; that is, emotional effects on EPN and LPP remain significant because of the high emotional intensity of the pictures. If this is true, then manipulations of spatial attention and perceptual load ought to reduce emotional effects on EPN and LPP more strongly for medium emotional pictures than for intense emotional pictures. However, no previous study has directly compared the effects of spatial inattention and perceptual load on responses to emotional pictures at fixation that vary in emotional intensity.

In the present study, IAPS pictures were selected that had three levels of emotional intensity: high negative, medium negative, and neutral pictures. Spatial attention and perceptual load were manipulated by four tasks that participants performed in separate blocks. On each trial of all tasks, an IAPS picture at the center of the screen was shown together with a six-letter string that covered a small section in the middle of the picture. In the picture task, participants attended the pictures (rather than the letter string) and were to respond only if the picture was shown in black and white $(25 \%$ of trials). As a manipulation of spatial attention, the other tasks required participants to attend the letter string (rather than the picture) and to respond only if the letter string contained either $\mathrm{N}$ or $\mathrm{X}$ ( $25 \%$ of trials). In the 1-letter task, the letter string consisted only of a single letter and five fillers. Because the picture task and the 1-letter task were simplemanipulating only whether participants attended the pictures or the letters - comparing responses between the tasks allowed us to study the effects of spatial attention. Accordingly, we expected that if high negative valence protects against spatial inattention, then the decrease in emotional effects on EPN and LPP between the picture task and the 1-letter task would be greater for medium negative pictures than for high negative pictures. As a manipulation of perceptual load, participants performed different letter tasks that varied the number of relevant letters - that is, either three letters and three fillers (3letter task) or six letters (6-letter task). So, comparing responses between the 1-letter, 3-letter, and 6-letter task allowed us to study the effects of perceptual load. Accordingly, we expected that if high negative valence protects against perceptual load, then emotional effects on EPN and LPP would decrease more strongly for medium negative than for high negative pictures with increased perceptual load. Because trials that required a response differed physically between tasks (e.g., picture in black and white vs. color), only the trials that did not prompt a response (i.e., 75\%) were analyzed, to avoid confounds on ERPs.

\section{Method}

Participants were 26 undergraduate students (12 women, mean age $=25.23, S D=4.89$, range 19-40) from universities in Stockholm, Sweden. Participation was based on informed consent and was rewarded with course credits or movie vouchers. The research was approved by the regional ethics board.

From the IAPS set (Lang et al., 2008), 180 color pictures were selected to vary from neutral valence and low arousal to negative valence and high arousal. ${ }^{1}$ Thus, the effects of high arousal and negative valence cannot be separated in

\footnotetext{
${ }^{1}$ The codes for the IAPS pictures were as follows: Neutral (1670, 1903, 1947, 2018, 2025, 2032, 2101, 2102, 2104, 2190, 2191, 2210, $2214,2220,2221,2273,2305,2359,2372,2377,2383,2384,2411$, $2445,2446,2484,2488,2489,2513,2514,2521,2575,2635,4613$, 5390, 6150, 7003, 7009, 7014, 7026, 7030, 7031, 7052, 7057, 7061, 7080, 7090, 7150, 7170, 7192, 7233, 7287, 7490, 7512, 7513, 7547, $8312,8465,8620,9260)$, medium $(1019,1033,1052,1070,1090$, $1110,1200,1202,1205,1220,1274,1931,1932,2115,2120,2301$, 2457, 2661, 2692, 2715, 2752, 3190, 3213, 3360, 5961, 5973, 6020, $6200,6210,6220,6410,6530,6550,6561,6610,7023,7360,9005$, 9008, 9373, 9395, 9403, 9409, 9440, 9445, 9530, 9582, 9584, 9590, 9592, 9594, 9596, 9599, 9610, 9909, 9912, 9925, 9926, 9930, 9941), and high $(2703,2730,2800,2811,2981,3000,3001,3010,3019$, 3030, 3051, 3053, 3059, 3060, 3068, 3069, 3071, 3080, 3100, 3103, $3110,3120,3130,3131,3150,3195,3225,3230,3261,6021,6230$, $6313,6360,6510,6520,6540,6563,6570,9006,9040,9075,9140$, 9163, 9181, 9183, 9185, 9187, 9253, 9302, 9325, 9326, 9332, 9410, 9412, 9413, 9420, 9560, 9570, 9571, 9940).
} 
the current picture set. On the basis of the IAPS norms across gender, the pictures were grouped as neutral $(n=60)$ with a mean normative valence of $5.27(S D=0.46)$ and a mean normative arousal of $3.48(S D=0.63)$; as medium negative $(n=60)$ with a mean normative valence of 3.44 $(S D=0.39)$ and a mean normative arousal of $5.47(S D=$ $0.67)$; and as high negative $(n=60)$ with a mean normative valence of $1.96(S D=0.33)$ and a mean normative arousal of $6.36(S D=0.59)$. All pairwise comparisons ( $t$ tests) between picture groups were significant on valence and arousal $(p<.001)$.

Because picture composition (i.e., figure vs. scene) confounds EPN amplitudes (Wiens, Sand, \& Olofsson, 2011), we tried to match the three emotion levels on picture composition. Unfortunately, in the IAPS set, neutral pictures are mainly figures, and emotional pictures are mainly scenes. Therefore, we could match only the high and medium picture groups in their composition. As confirmed in a pilot study $(n=9)$ in which picture complexity was rated (from 1 = figure to $9=$ scene), an ANOVA (with individual pictures as subjects) showed that mean complexity ratings differed among the three picture categories, $F(2,174)=11.57, p<.001$. Neutral pictures received lower complexity ratings (2.67) than did medium pictures (3.66) and high pictures (3.53), whereas medium and high pictures did not differ $(p>.50)$. These results rule out picture composition as a potential confound for any different effects of medium and high pictures in subsequent analyses. Also, although picture composition differed between neutral pictures (figures) and emotional pictures (scenes), picture composition suppresses the EPN (Wiens, Sand, \& Olofsson, 2011). Therefore, any confounding effect on EPN would only reduce power in detecting differences between emotional and neutral pictures.

Visual stimuli were shown at a distance of $80 \mathrm{~cm}$ (chin rest) on a 21-in. View Sonic P227f cathode ray-tube monitor at a $100-\mathrm{Hz}$ refresh rate with a resolution of $1,280 \times 1,024$ pixels. Experiment software was Presentation 14.8 (Neurobehavioral Systems, Inc., Albany, CA). The IAPS pictures were shown in color at $19 \times 13.5 \mathrm{~cm}(13.5 \mathrm{x}$ $9.6^{\circ}$ visual angle) in landscape on a black background. Letters were $\mathrm{H}, \mathrm{K}, \mathrm{M}, \mathrm{N}, \mathrm{O}, \mathrm{V}, \mathrm{W}, \mathrm{X}$, and Z. Letters were shown in gray in capital Courier New Bold. Two rows of three letters were shown in the middle of the pictures within a black square of $2 \times 2 \mathrm{~cm}\left(1.4 \times 1.4^{\circ}\right.$ visual angle $)$.

The electroencephalogram (EEG) was recorded from 128 electrodes according to the $\mathrm{ABC}$ system (i.e., electrodes are arranged in circles with different radii from the vertex) with an Active Two Biosemi system (BioSemi, Amsterdam, Netherlands). The data were sampled at $512 \mathrm{~Hz}$ and were filtered only with a built-in low-pass filter at $104 \mathrm{~Hz}$ and an offline notch filter at $50 \mathrm{~Hz}$.
Procedure

Participants performed four tasks in separate blocks; task order was random over participants. Figure 1 illustrates the four tasks together with examples of picture stimuli. Each picture stimulus consisted of an IAPS picture at fixation and two rows of three letters in the middle of the picture. Each trial comprised a fixation cross (800-1,000 ms), the picture stimulus $(200 \mathrm{~ms})$, and a blank screen $(1,300 \mathrm{~ms})$.

In the picture task, participants were to ignore the letters and to press the space key only if the picture was shown in black and white. In the remaining three blocks, participants performed a letter task and were instructed to ignore the pictures and to press space only if the target letter $\mathrm{N}$ or $\mathrm{X}$ was shown. In the 1-letter task, five of the six letters were fillers (i.e., O), and only a single letter was either a distracter (i.e., H, K, M, V, W, or Z) or a target letter (i.e., N or X). In the 3-letter task, three of the six letters were fillers, and the remaining three letters were either three distracters or two distracters and a target. In the 6-letter task, the six letters were either all distracters or five distracters and a target letter. At the beginning of each task, participants were instructed to keep their gaze on the center of the screen (i.e., position of fixation cross) throughout the task and to respond as quickly as possible while minimizing errors.

Each task used the 180 IAPS pictures once in separate trials. Of the 180 trials, 45 were target trials $(25 \%)$ that required participants to push the space key because of a black-and-white picture during the picture task, or the letter $\mathrm{N}$ or $\mathrm{X}$ during the letter tasks. Of the 60 pictures in each emotion picture group (high, medium, neutral), 15 were selected randomly on each task to serve as targets and 45 as nontargets. In each task, trial order was random with the restriction that 1 of 4 trials was a target trial.

Regarding letter selection, distracters and targets on each trial were drawn randomly from the distracter and target sets (without replacement), and the positions of the six letters within the two rows of three letters were random. In the picture task, one-third of the trials each had one, three, or six distracters to resemble the stimulus conditions during the different letter tasks.

To familiarize participants with the tasks, participants could complete 24 practice trials before each task with pictures different from those used in the actual task. The same pictures were used in all practice tasks.

\section{Data reduction}

In each task, 45 trials were target trials and required participants to push the space key. Of these 45 trials, 15 each depicted high, medium, and neutral pictures. These 


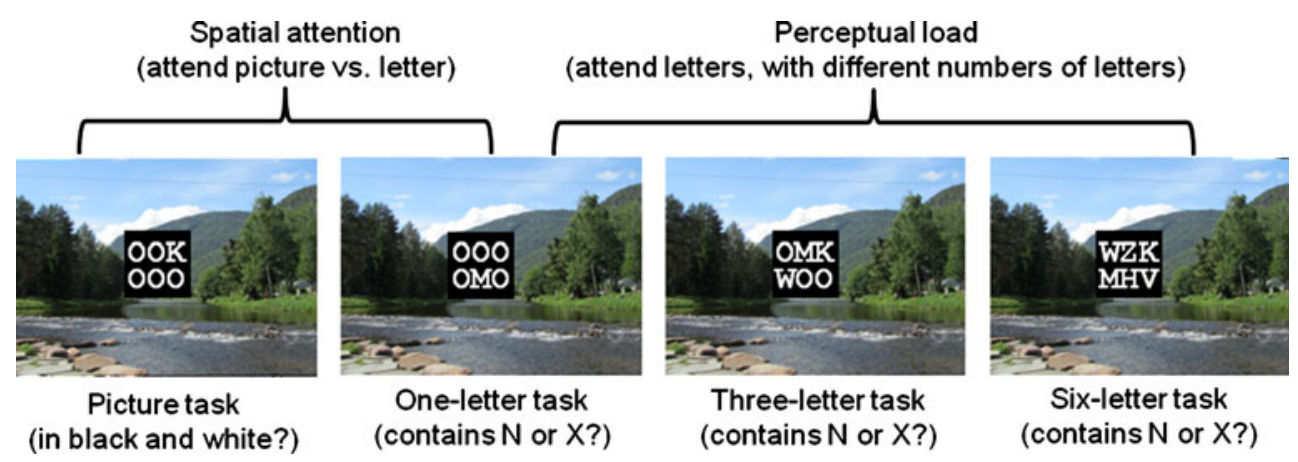

Fig. 1 Illustration of the four different tasks together with examples of picture stimuli. The comparison between the picture task and the 1letter task captures the manipulation of directed (spatial) attention, and the comparison among the 1-letter, 3-letter, and 6-letter tasks captures the manipulation of perceptual load. On each trial, the picture stimulus consisted of an IAPS picture with two rows of three letters in the center (not drawn to scale). In the picture task, participants were to ignore the letters and to respond only if the picture was shown in black and white. In the remaining three tasks, participants were to

data were used to compute hit rates and reaction times (RTs) to hits. In preliminary analyses, the false-alarm rates on nontarget trials were used to compute $d$ ' for signal detection analyses. Because results were comparable for hit rates and $d$, we report the more intuitive results for hit rates.

The software BESA (Version 5.3.7, MEGIS Software $\mathrm{GmbH}$, Gräfelfing, Germany, www.BESA.de) was used for offline processing of the EEG. Noisy electrodes (maximum of six for 12 participants) were interpolated with spherical splines. Eye blinks were corrected with a built-in algorithm (15 surrogate brain sources). Only nontarget trials without button presses were analyzed to reduce confounding effects from motor responses and differences in physical features (i.e., picture in black and white or in color). Epochs ranged from $100 \mathrm{~ms}$ before to $1,300 \mathrm{~ms}$ after stimulus onset. Epochs were baseline corrected $(100 \mathrm{~ms})$ and re-referenced to the arithmetic average of all electrodes.

Across participants, the ERP difference waves to the negative (high and medium combined) minus neutral pictures were used to identify, through visual inspection, electrode clusters and intervals that captured emotional effects on EPN and LPP. In these analyses, the ERPs were collapsed across tasks to detect the main effect of emotion (i.e., negative minus neutral). Across tasks, a greater negativity to negative (high and medium combined) versus neutral (i.e., the EPN) pictures was evident between 200 and $280 \mathrm{~ms}$ across six electrodes (A13, A14, and A24A27), and a greater positivity to negative (high and medium combined) versus neutral pictures (i.e., the LPP) was strongest between $400-700 \mathrm{~ms}$ across eight electrodes (A01-A04, B01-B02, D15-D16). For each participant, the mean amplitudes (in $\mu \mathrm{V}$ ) for the relevant electrodes and intervals were extracted for each trial. Then, these ampli- ignore the pictures and to respond only if the target letter $\mathrm{N}$ or $\mathrm{X}$ was shown. In the 1-letter task, five of the six letters were fillers (i.e., O), and only a single letter was either a distracter (i.e., H, K, M, V, W, or $\mathrm{Z}$ ) or a target letter (i.e., $\mathrm{N}$ or $\mathrm{X}$ ). In the 3-letter task, three of the six letters were fillers, and the remaining three letters were either three distracters or two distracters and a target. In the 6-letter task, the six letters were either all distracters or five distracters and a target letter. The probability for a target trial was $25 \%$. Note that ERP data were analyzed only for nontarget trials without a button press

tudes for EPN and LPP were averaged separately for each emotion and task.

To study effects of shifting spatial attention from the pictures onto the letters on behavioral data (hit rates and RT to hits) and mean amplitudes, the first ANOVA ( $3 \times 2)$ included the within-subjects variables emotion (high, medium, neutral) and task (picture, 1-letter). To study effects of perceptual load, the second ANOVA ( $3 \times 3$ ) included the within-subjects variables emotion (high, medium, neutral) and task (picture, 1-letter, 3-letter, 6-letter). Because in preliminary analyses, gender and task order had no effects, the simpler ANOVAs without these variables are reported below. Follow up ANOVAs and $t$ tests were conducted to evaluate specific effects of emotion (e.g., high vs. medium) across tasks. Results of the ANOVAs are reported with uncorrected $d f$ values but with GreenhouseGeisser corrected $F$ values and significance levels that were considered significant at two-tailed $p<.05$.

\section{Results}

Hit rates and reaction time

Figure 2 shows mean hit rates separately for the three emotion levels and the four task levels. In regards to effects of spatial attention, the ANOVA ( $3 \times 2)$ with emotion (high, medium, and neutral) and task (picture, 1-letter) showed an interaction between task and emotion, $F(2,50)=3.89, p=$ $.036, \eta_{p}^{2}=.13$. There was also an effect of task, $F(1,50)=$ $10.73, p=.003, \eta_{p}^{2}=.30$, but only a trend for an effect of emotion, $F(2,50)=3.14, p=.052, \eta_{p}^{2}=.11$. As is shown 


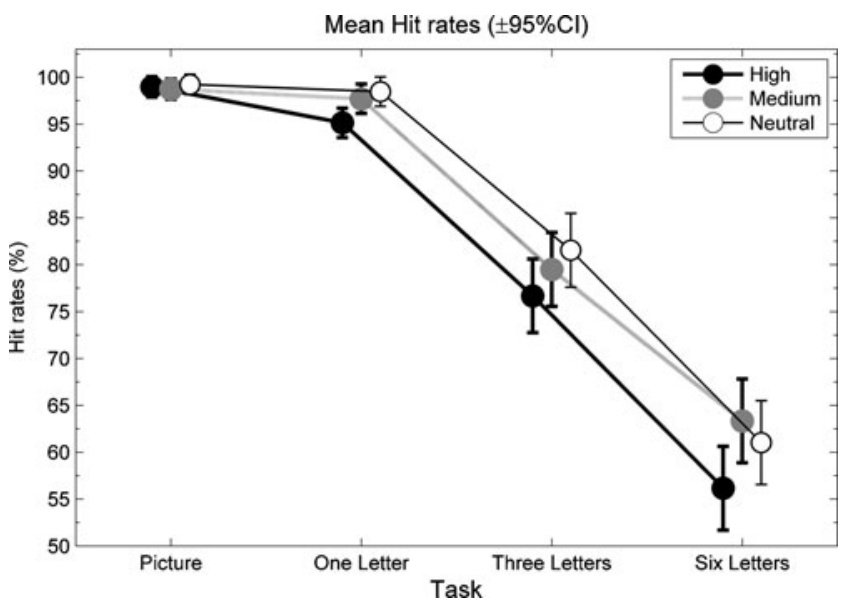

Fig. 2 Mean hit rates (in \%) for high, medium, and neutral pictures during each task. The error bars refer to the $95 \%$ CI from the pooled error term of the ANOVA for each task

in Fig. 2, the interaction suggested that during the picture task, performance showed a clear ceiling effect on all pictures, whereas during the 1-letter task, performance was lower to high pictures than to medium and neutral pictures.

In regard to effects of perceptual load, the ANOVA $(3 \times 3)$ with emotion (high, medium, and neutral) and task (1-letter, 3letter, 6-letter) showed no interaction $(p<.50)$, but effects of emotion, $F(2,50)=5.46, p=.013, \eta_{p}^{2}=.18$, and task, $F(2,50)=117.51, p<.001, \eta_{p}^{2}=.82$. Across the letter tasks, performance was lower for high than for medium pictures and for high than for neutral pictures ( $p<.05$ on $t$ tests), but did not differ between medium and neutral pictures $(p>.90)$. Also, performance decreased substantially with more letters ( $p<.001$ for neighboring tasks). Similar analyses of RTs to hits showed only effects of task $(p<.001)$ so that RTs increased gradually from the picture task to the 6-letter task.

\section{Early posterior negativity (EPN)}

Figure 3 shows the results for the EPN. Regarding effects of spatial attention, the ANOVA $(3 \times 2)$ with emotion (high, medium, and neutral) and task (picture, 1-letter) yielded only a trend for an interaction, $F(2,50)=2.56, p=.09, \eta_{p}^{2}=.09$, but significant effects of emotion, $F(2,50)=11.76, p<.001$, $\eta_{p}^{2}=.32$, and task, $F(1,25)=30.37, p<.001, \eta_{p}^{2}=.55$. Similarly, the focused interaction between high and medium pictures for the picture task versus 1-letter task showed only a trend, $F(1,25)=4.14, p=.053, \eta_{p}^{2}=.14$. Although tentative, these results suggested that the amplitude difference between high and medium pictures during the picture task was attenuated during the 1-letter task.

Regarding effects of perceptual load, the ANOVA ( $3 \times 3)$ with emotion (high, medium, and neutral) and task (1-letter, 3-letter, 6-letter) showed no interaction of emotion and task
( $p>.70)$, but an effect of emotion, $F(2,50)=8.60, p<$ $.001, \eta_{p}^{2}=.26$. Across tasks, amplitudes were lower for high than for neutral pictures and for medium than for neutral pictures ( $p<.001$ on $t$ tests) but not for high than for medium pictures $(p>.40)$.

Late positive potential (LPP)

Figure 4 shows the results for the LPP. Regarding spatial attention, the ANOVA ( $3 \times 2$ ) with emotion and task (picture, 1-letter) yielded an interaction, $F(2,50)=$ 13.39, $p<.001, \eta_{p}^{2}=.35$, and significant effects of emotion, $F(2,50)=13.93, p<.001, \eta_{p}^{2}=.36$, and task, $F(1,25)=6.65, p=.016, \eta_{p}^{2}=.21$. Also, the specific interaction between high and medium pictures for the picture task versus 1-letter task was significant, $F(1,25)=$ 4.83, $p=.037, \eta_{p}^{2}=.16$. As is shown in Fig. 4, the difference in LPP between high and medium emotional pictures decreased from the picture task to the 1-letter task.

Regarding perceptual load, the ANOVA ( $3 \times 3)$ with emotion and task (1-letter, 3-letter, 6-letter) suggested no interaction between emotion and task $(p>.60)$ and no effect of task $(p>.30)$, but an effect of emotion, $F(2,50)=$ 3.94, $p=.026, \eta_{p}^{2}=.14$. Across tasks, amplitudes were larger for high than for neutral pictures and for medium than for neutral pictures $(p<.05$ on $t$ tests), but did not differ between high and medium pictures $(p>.80)$.

\section{Discussion}

With regard to the effects of spatial inattention, the main finding was that the difference in LPP to high versus medium negative pictures decreased from when participants attended the pictures (during the picture task) to when they attended the letters (during the 1-letter task). With regard to the effects of perceptual load, the main finding was that EPN and LPP were similar for high and medium negative pictures over the three letter tasks (1-letter, 3-letter, and 6letter task). In contrast, emotional effects on hit rates were observed only for high negative pictures.

The shift of spatial attention from the pictures (during the picture task) to the letters (during the 1-letter task) decreased the difference in LPP to high versus medium negative pictures. As is shown in Fig. 4, these findings suggest that spatial inattention eliminated the difference in LPP between high and medium negative pictures. However, this pattern of results alone does not allow one to decide whether spatial inattention decreased the LPP to high negative pictures or whether spatial inattention increased the LPP to medium negative pictures. To resolve this question, the LPP to neutral pictures needs to serve as a baseline, because emotional 
Fig. 3 The top row shows the mean ERP waves for high, medium, and neutral pictures during each task across the relevant electrodes for the early posterior negativity (EPN). Data were referenced to the average of all 128 electrodes. Because emotional effects are small as compared with the overall size of the ERPs, the middle row shows blow-ups of the relevant intervals. Furthermore, the bottom row shows the mean amplitudes across $200-280 \mathrm{~ms}$ separately for the three picture groups and the four tasks across the six relevant electrodes. The error bars refer to the $95 \% \mathrm{CI}$ from the pooled error term of the ANOVA for each task. Last, the inset shows the topography of the main effect of emotion (high and medium vs. neutral) across all tasks. The relevant electrodes in computing the mean amplitudes are indicated by their labels
EPN
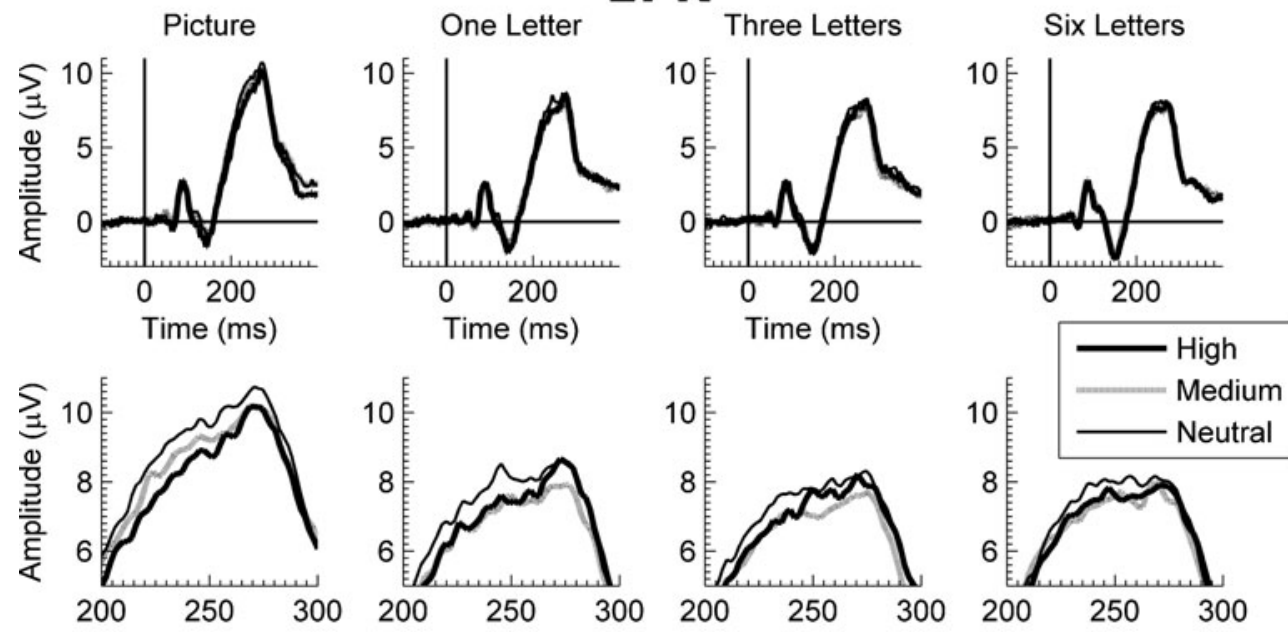

Mean EPN amplitude (200-280 ms; $\pm 95 \% \mathrm{Cl})$

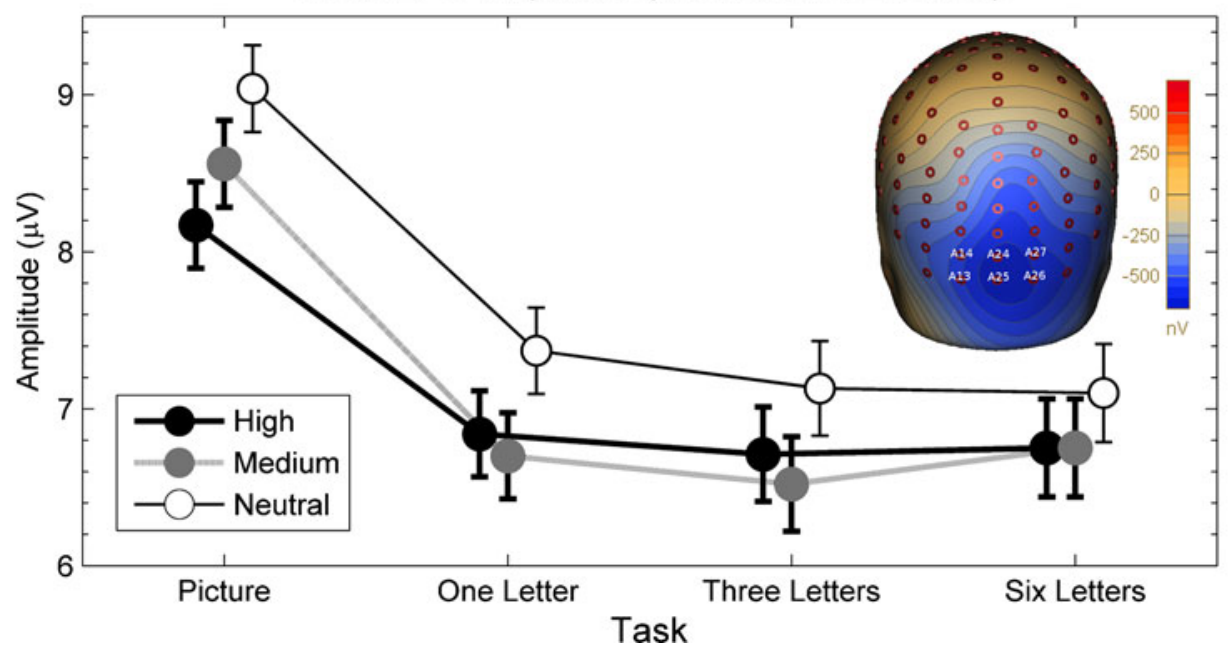

effects on LPP are derived from difference scores of LPPs to emotional versus neutral pictures (Schupp et al., 2006). Thus, the decrease in difference scores between LPP to emotional versus neutral pictures was greater for the high negative pictures than for the medium negative pictures between the picture task and the 1-letter task. ${ }^{2}$ As is shown in Fig. 3, the EPN showed a similar trend in that the decrease in difference scores between EPN to emotional versus neutral pictures tended to be greater for the high negative pictures than for the medium negative pictures between the

\footnotetext{
$\overline{2}$ The pattern of results in Fig. 4 suggests that LPP amplitudes to high negative pictures remained stable, whereas LPP amplitudes to neutral pictures and medium negative pictures increased from the picture task to the 1-letter task. However, the pattern of results from other studies favors our interpretation in that LPP amplitudes to emotional pictures decreased, whereas responses to neutral pictures remained relatively stable (Sand \& Wiens, 2011; Wiens, Sand, Norberg, \& Andersson, 2011). Accordingly, a parsimonious explanation is that the letter task generally increased LPP amplitudes to all pictures (i.e., main effect of task), but that the reduced emotional processing of high and medium negative pictures cancelled out the increase in LPP amplitudes from the task effect (i.e., interaction between task and emotion).
}

picture task and the 1-letter task. This result of only a trend level effect of spatial inattention on EPN suggests that the effect may be relatively weak, which might explain why one of our previous studies found significant results (Wiens, Sand, Norberg et al., 2011), whereas the other did not (Sand \& Wiens, 2011). Furthermore, the finding that spatial inattention generally reduced emotional effects on LPP to emotional versus neutral pictures replicates previous results (Sand \& Wiens, 2011; Wiens, Sand, Norberg et al., 2011). Critically, our results suggest that spatial inattention reduced emotional effects on LPP (and EPN) more strongly for high than for medium negative pictures. Therefore, our results are not consistent with the prediction that high emotional intensity protects more against manipulations of spatial attention than does medium emotional intensity, which would have resulted in a stronger reduction of emotional effects on EPN and LPP for medium than high negative pictures.

As is illustrated in Figs. 3 and 4, the manipulation of perceptual load (from 1-letter task to 6-letter task) showed that EPN and LPP were similar for high and medium 
Fig. 4 The top row shows the mean ERP waves for high, medium, and neutral pictures during each task across the relevant electrodes for the late positive potential (LPP). Data were referenced to the average of all 128 electrodes. Because emotional effects are small as compared with the overall size of the ERPs, the middle row shows blow-ups of the relevant intervals. Furthermore, the bottom row shows the mean amplitudes across 400-700 ms separately for the three picture groups and the four tasks across the eight relevant electrodes.

The error bars refer to the $95 \%$ $\mathrm{CI}$ from the pooled error term of the ANOVA for each task. Last, the inset shows the topography of the main effect of emotion (high and medium vs. neutral) across all tasks. The relevant electrodes in computing the mean amplitudes are indicated by their labels
LPP
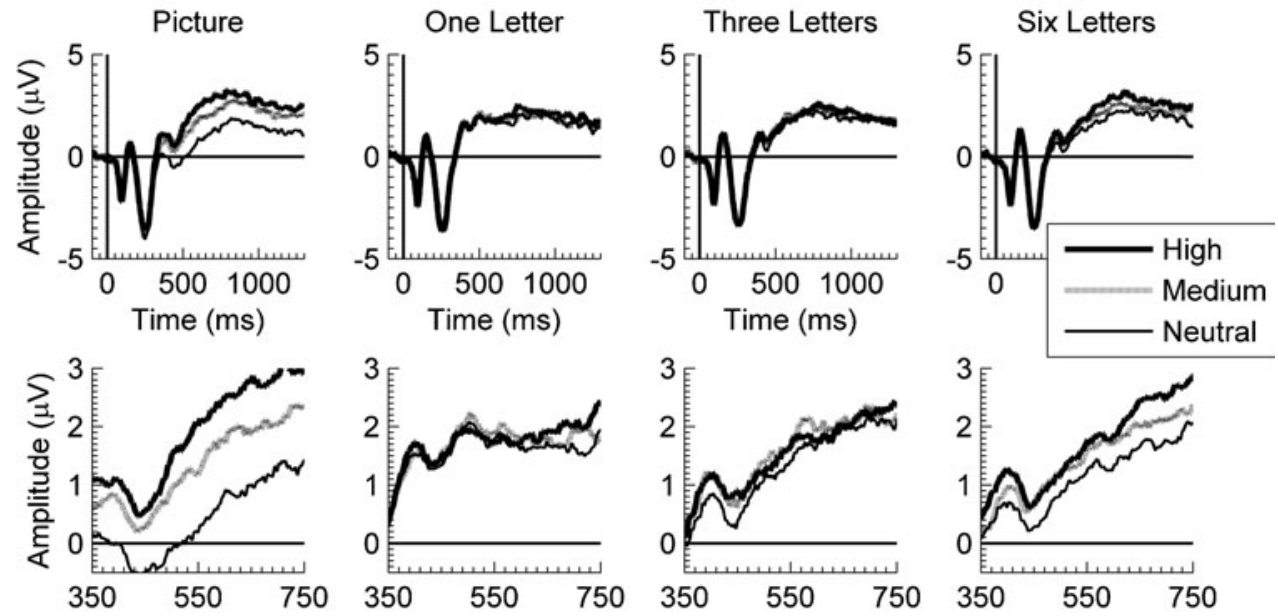

Mean LPP amplitude $(400-700 \mathrm{~ms} ; \pm 95 \% \mathrm{Cl})$

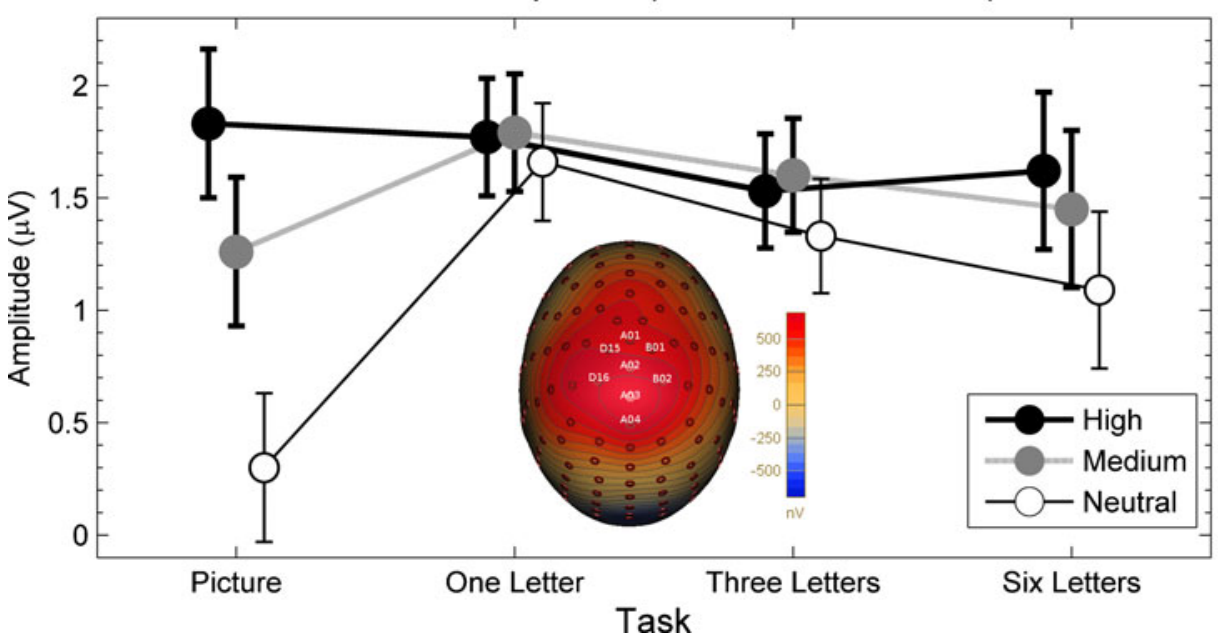

negative pictures in the three letter tasks (1-letter, 3-letter, and 6-letter task). As a manipulation check of perceptual load, the behavioral results showed that hit rates strongly decreased and RTs to hits strongly increased from the 1-letter task to the 6-letter task (see Fig. 2). These findings rule out a speed-accuracy trade-off between the different letter tasks. Instead, they indicate that perceptual load was increased strongly by the number of letters that participants had to process (Lavie, 1995, 2010). Critically, despite its substantial behavioral effects, perceptual load had no effect on EPN and LPP. These null findings are consistent with those of previous studies, which also failed to detect effects of perceptual load on EPN and LPP (Norberg et al., 2010; Sand \& Wiens, 2011). Notably, although EPN and LPP amplitudes in the letter tasks were similar to high negative pictures and medium negative pictures, both differed from the EPN and LPP amplitudes to neutral pictures. As is shown in Fig. 3, the EPN amplitudes to high and medium negative pictures were less positive than the EPN amplitudes to neutral pictures; thus, there were clear emotional effects on EPN for both high and medium negative pictures. As is shown in Fig. 4, the LPP amplitudes to high and medium negative pictures were more positive than the LPP amplitudes to neutral pictures; thus, there were clear emotional effects on LPP for both high and medium negative pictures. Because individual emotional effects on EPN and LPP were significant for both high negative and medium negative pictures, the observed null findings for differential effects of perceptual load between high negative and medium negative pictures were probably not caused by a lack of power.

In sum, our results suggest that effects of perceptual load on EPN and LPP are similar for high negative and medium negative pictures. As for the effects of spatial attention, our results do not support the prediction that high emotional intensity protects more against manipulations of perceptual load than does medium emotional intensity, which would have resulted in a stronger reduction of emotional effects on EPN and LPP for medium than high negative pictures.

This conclusion seems valid with regard to ERPs; however, it cannot be generalized to behavioral effects. Across the letter tasks, hit rates were lower to high negative than to either 
medium negative or neutral pictures, which did not differ from each other. So, although the ERP results did not support a particular resistance to perceptual load for high negative pictures, the behavioral evidence showed a difference between high negative and medium negative pictures so that distracting effects on hit rates remained significant for high negative pictures irrespective of perceptual load. Although these findings are not consistent with a previous report that behavioral effects from negative pictures at fixation were eliminated under perceptual load (Okon-Singer, Tzelgov, \& Henik, 2007), they are consistent with other studies that found that ERP and behavioral measures can differ in their sensitivity in detecting effects (MacNamara \& Hajcak, 2009, 2010). These behavioral results imply that ERP measures cannot be viewed as exhaustive measures of emotional effects on attention and performance and support the complementary nature of ERP and behavioral measures.

To conclude, our results do not support the prediction that, in comparison with medium emotional pictures, high emotional pictures at fixation protect indices of motivated attention (EPN, LPP) from manipulations of spatial attention and perceptual load. Rather, when pictures were not spatially attended, the difference scores between LPP to emotional versus neutral pictures decreased more strongly for high negative than for medium negative pictures. Also, when perceptual load was manipulated, emotional effects on EPN and LPP were similar for high negative and medium negative pictures. In fact, because the residual emotional effects on EPN and LPP were similar for high and medium negative pictures, these results imply that for emotional pictures at fixation, a medium level of emotional intensity is sufficient to protect indexes of motivated attention (EPN, LPP) from manipulations of spatial attention and perceptual load.

Author Note This research was supported by grants 421-2007-2606 and 421-2010-1697 from the Swedish Research Council to Stefan Wiens. The authors thank Stephen Palmer for editorial assistance. The authors declare no conflict of interest. Address correspondence to S. Wiens, Department of Psychology, Frescati Hagväg 9, rum 219 (växthus B), Stockholm University, 10691 Stockholm, Sweden (e-mail: sws@psychology.su.se).

\section{References}

Bradley, M. M. (2009). Natural selective attention: Orienting and emotion. Psychophysiology, 46, 1-11. doi:10.1111/j.14698986.2008.00702.x

Britton, J. C., Taylor, S. F., Sudheimer, K. D., \& Liberzon, I. (2006). Facial expressions and complex IAPS pictures: Common and differential networks. NeuroImage, 31, 906-919. doi:10.1016/j. neuroimage.2005.12.050

Codispoti, M., Ferrari, V., De Cesarei, A., \& Cardinale, R. (2006). Implicit and explicit categorization of natural scenes. Progress in Brain Research, 156, 53-65.
Cuthbert, B. N., Schupp, H. T., Bradley, M. M., Birbaumer, N., \& Lang, P. J. (2000). Brain potentials in affective picture processing: Covariation with autonomic arousal and affective report. Biological Psychology, 52, 95-111.

De Cesarei, A., Codispoti, M., \& Schupp, H. T. (2009). Peripheral vision and preferential emotion processing. NeuroReport, 20, 1439-1443. doi:10.1097/WNR.0b013e3283317d3e

Dunning, J. P., \& Hajcak, G. (2009). See no evil: Directing visual attention within unpleasant images modulates the electrocortical response. Psychophysiology, 46, 28-33. doi:10.1111/j.14698986.2008.00723.x

Eimer, M., Holmes, A., \& McGlone, F. P. (2003). The role of spatial attention in the processing of facial expression: An ERP study of rapid brain responses to six basic emotions. Cognitive, Affective, \& Behavioral Neuroscience, 3, 97-110.

Foti, D., Hajcak, G., \& Dien, J. (2009). Differentiating neural responses to emotional pictures: Evidence from temporal-spatial PCA. Psychophysiology, 46, 521-530. doi:10.1111/j.14698986.2009.00796.x

Hajcak, G., Dunning, J. P., \& Foti, D. (2007). Neural response to emotional pictures is unaffected by concurrent task difficulty: An event-related potential study. Behavioral Neuroscience, 121, 1156-1162. doi:10.1037/0735-7044.121.6.1156

Hajcak, G., Dunning, J. P., \& Foti, D. (2009). Motivated and controlled attention to emotion: Time-course of the late positive potential. Clinical Neurophysiology, 120, 505-510. doi:10.1016/ j.clinph.2008.11.028

Hajcak, G., MacNamara, A., \& Olvet, D. M. (2010). Event-related potentials, emotion, and emotion regulation: An integrative review. Developmental Neuropsychology, 35, 129-155.

Holmes, A., Kiss, M., \& Eimer, M. (2006). Attention modulates the processing of emotional expression triggered by foveal faces. Neuroscience Letters, 394, 48-52.

Holmes, A., Nielsen, M. K., Tipper, S., \& Green, S. (2009). An electrophysiological investigation into the automaticity of emotional face processing in high versus low trait anxious individuals. Cognitive, Affective, \& Behavioral Neuroscience, 9, 323-334. doi:10.3758/cabn.9.3.323

Holmes, A., Vuilleumier, P., \& Eimer, M. (2003). The processing of emotional facial expression is gated by spatial attention: Evidence from event-related brain potentials. Cognitive Brain Research, 16, 174-184.

Junghöfer, M., Bradley, M. M., Elbert, T. R., \& Lang, P. J. (2001). Fleeting images: A new look at early emotion discrimination. Psychophysiology, 38, 175-178.

Kissler, J., Herbert, C., Winkler, I., \& Junghöfer, M. (2009). Emotion and attention in visual word processing-An ERP study. Biological Psychology, 80, 75-83. doi:10.1016/j.biopsycho.2008.03.004

Lang, P. J., Bradley, M. M., \& Cuthbert, B. N. (2008). International affective picture system (IAPS): Affective ratings of pictures and instruction manual. Technical Report A-8. Gainesville, FL: University of Florida.

Lavie, N. (1995). Perceptual load as a necessary condition for selective attention. Journal of Experimental Psychology. Human Perception and Performance, 21, 451-468.

Lavie, N. (2010). Attention, distraction, and cognitive control under load. Current Directions in Psychological Science, 19, 143-148. doi:10.1177/0963721410370295

MacNamara, A., Ferri, J., \& Hajcak, G. (2011). Working memory load reduces the late positive potential and this effect is attenuated with increasing anxiety. Cognitive, Affective, \& Behavioral Neuroscience, 11, 321-331. doi:10.3758/s13415-011-0036-z

MacNamara, A., \& Hajcak, G. (2009). Anxiety and spatial attention moderate the electrocortical response to aversive pictures. Neuropsychologia, 47, 2975-2980. doi:10.1016/j. neuropsychologia.2009.06.026 
MacNamara, A., \& Hajcak, G. (2010). Distinct electrocortical and behavioral evidence for increased attention to threat in generalized anxiety disorder. Depression and Anxiety, 27, 234-243. doi:10.1002/da.20679

Mocaiber, I., Pereira, M. G., Erthal, F. S., Machado-Pinheiro, W., David, I. A., \& Cagy, M. (2010). Fact or fiction? An eventrelated potential study of implicit emotion regulation. Neuroscience Letters, 476, 84-88. doi:10.1016/j.neulet.2010.04.008

Norberg, J., Peira, N., \& Wiens, S. (2010). Never mind the spider: Late positive potentials to phobic threat at fixation are unaffected by perceptual load. Psychophysiology, 47, 1151-1158. doi:10.1111/j.1469-8986.2010.01019.x

Okon-Singer, H., Tzelgov, J., \& Henik, A. (2007). Distinguishing between automaticity and attention in the processing of emotionally significant stimuli. Emotion, 7, 147-157.

Olofsson, J. K., Nordin, S., Sequeira, H., \& Polich, J. (2008). Affective picture processing: An integrative review of ERP findings. Biological Psychology, 77, 247-265. doi:10.1016/j. biopsycho.2007.11.006

Palomba, D., Angrilli, A., \& Mini, A. (1997). Visual evoked potentials, heart rate responses and memory to emotional pictorial stimuli. International Journal of Psychophysiology, 27, 55-67.

Peira, N., Golkar, A., Larsson, M., \& Wiens, S. (2010). What you fear will appear: Detection of schematic spiders in spider fear. Experimental Psychology, 57, 470-475.

Pessoa, L. (2005). To what extent are emotional visual stimuli processed without attention and awareness? Current Opinion in Neurobiology, 15, 188-196.

Pessoa, L., Padmala, S., \& Morland, T. (2005). Fate of unattended fearful faces in the amygdala is determined by both attentional resources and cognitive modulation. NeuroImage, 28, 249-255.

Sabatinelli, D., Lang, P. J., Keil, A., \& Bradley, M. M. (2007). Emotional perception: Correlation of functional MRI and eventrelated potentials. Cerebral Cortex, 17, 1085-1091.
Sand, A., \& Wiens, S. (2011). Processing of unattended, simple negative pictures resists perceptual load. NeuroReport, 22, 348-352. doi:10.1097/WNR.0b013e3283463cb1

Schupp, H. T., Flaisch, T., Stockburger, J., \& Junghöfer, M. (2006). Emotion and attention: Event-related brain potential studies. In S. Anders, G. Ende, M. Junghöfer, J. Kissler, \& D. Wildgruber (Eds.), Progress in brain research: Understanding emotions, vol. 156 (pp. 31-51). Amsterdam, the Netherlands: Elsevier.

Schupp, H. T., Junghöfer, M., Weike, A. I., \& Hamm, A. O. (2003). Emotional facilitation of sensory processing in the visual cortex. Psychological Science, 14, 7-13.

Silvert, L., Lepsien, J., Fragopanagos, N., Goolsby, B., Kiss, M., \& Taylor, J. G. (2007). Influence of attentional demands on the processing of emotional facial expressions in the amygdala. NeuroImage, 38, 357-366.

Sutton, S., Braren, M., Zubin, J., \& John, E. R. (1965). Evoked-potential correlates of stimulus uncertainty. Science, 150, 1187-1188.

Vuilleumier, P. (2005). How brains beware: Neural mechanisms of emotional attention. Trends in Cognitive Sciences, 9, 585-594.

Vuilleumier, P., Armony, J. L., Driver, J., \& Dolan, R. J. (2001). Effects of attention and emotion on face processing in the human brain: An event-related fMRI study. Neuron, 30, 829-841.

Vuilleumier, P., \& Huang, Y. M. (2009). Emotional attention: Uncovering the mechanisms of affective biases in perception. Current Directions in Psychological Science, 18, 148-152.

Wiens, S., Sand, A., Norberg, J., \& Andersson, P. (2011a). Emotional event-related potentials are reduced if negative pictures presented at fixation are unattended. Neuroscience Letters, 495, 178-182. doi:DOI:10.1016/j.neulet.2011.03.042

Wiens, S., Sand, A., \& Olofsson, J. K. (2011b). Nonemotional features suppress early and enhance late emotional electrocortical responses to negative pictures. Biological Psychology, $86,83-89$. 